\title{
Particle swarm optimizer for variable weighting in clustering high-dimensional data
}

\author{
Yanping Lu • Shengrui Wang • Shaozi Li · Changle Zhou
}

Received: 30 June 2008 / Revised: 20 August 2009 / Accepted: 10 October 2009 /

Published online: 13 November 2009

(C) The Author(s) 2009

\begin{abstract}
In this paper, we present a particle swarm optimizer (PSO) to solve the variable weighting problem in projected clustering of high-dimensional data. Many subspace clustering algorithms fail to yield good cluster quality because they do not employ an efficient search strategy. In this paper, we are interested in soft projected clustering. We design a suitable $k$-means objective weighting function, in which a change of variable weights is exponentially reflected. We also transform the original constrained variable weighting problem into a problem with bound constraints, using a normalized representation of variable weights, and we utilize a particle swarm optimizer to minimize the objective function in order to search for global optima to the variable weighting problem in clustering. Our experimental results on both synthetic and real data show that the proposed algorithm greatly improves cluster quality. In addition, the results of the new algorithm are much less dependent on the initial cluster centroids. In an application to text clustering, we show that the algorithm can be easily adapted to other similarity measures, such as the extended Jaccard coefficient for text data, and can be very effective.
\end{abstract}

Keywords High-dimensional data P Projected clustering · Variable weighting · Particle swarm optimization $\cdot$ Text clustering

\section{Introduction}

Clustering high-dimensional data is a common task in various data mining applications. Text clustering is a typical example. In text mining, a text dataset is viewed as a matrix,

Editors: D. Martens, B. Baesens, and T. Fawcett.

Y. Lu (凶) · S. Wang

Department of Computer Science, University of Sherbrooke, 2500 Boul. de l'Universite, Sherbrooke, Quebec, J1K 2R1, Canada

e-mail: Yanping.Lu@USherbrooke.ca

Y. Lu $\cdot$ S. Li $\cdot$ C. Zhou

Department of Cognitive Science, Xiamen University, Xiamen, 361005, China 
where a row represents a document and a column represents a unique term. The number of dimensions is represented by the number of unique terms, which is usually in the thousands. Another application where high-dimensional data occurs is insurance company customer prediction. It is important to separate customers into groups to help companies predict who would be interested in buying an insurance policy (van der Putten and Someren 2000). Many other applications, such as bankruptcy prediction, web mining, protein function prediction, etc., present similar data analysis problems.

Clustering high-dimensional data is a difficult task because clusters of high-dimensional data are usually embedded in lower-dimensional subspaces. Traditional clustering algorithms struggle with high-dimensional data because the quality of results deteriorates due to the curse of dimensionality. As the number of dimensions increases, data becomes very sparse and distance measures in the whole dimension space become meaningless. In fact, some dimensions may be irrelevant or redundant for clusters and different sets of dimensions may be relevant for different clusters. Thus, clusters should often be searched for in subspaces of dimensions rather than the whole dimension space.

According to the definition of the problem, the approaches to identify clusters on such datasets are categorized into three types (Kriegel et al. 2009). The first, subspace clustering, aims to identify clusters in all subspaces of the entire feature space (see for instance Agrawal et al. 2005; Goil et al. 1999; Moise and Sander 2008). The second, projected clustering, aims to partition the dataset into disjoint clusters (see for instance Procopiuc et al. 2002; Woo et al. 2004). The third type, hybrid clustering, includes algorithms that fall into neither of the first two types (see for instance Elke et al. 2008; Moise et al. 2008). The performance of many subspace/projected clustering algorithms drops quickly with the size of the subspaces in which the clusters are found (Parsons et al. 2004). Also, many of them require domain knowledge provided by the user to help select and tune their settings, such as the maximum distance between dimensional values (Procopiuc et al. 2002), the threshold of input parameters (Elke et al. 2008; Moise et al. 2008) and the density (Agrawal et al. 2005; Goil et al. 1999; Moise and Sander 2008), which are difficult to set.

Recently, soft projected clustering, a special case of projected clustering, has been developed to identify clusters by assigning an optimal variable weight vector to each cluster (Domeniconi et al. 2007; Huang et al. 2005; Jing et al. 2007). Each of these algorithms minimizes a $k$-means like objective function iteratively. Although the cluster membership of an object is calculated in the whole variable space, the similarity between each pair of objects is based on weighted variable differences. The variable weights transform the Euclidean distance used in conventional $k$-means algorithm so that the associated cluster is reshaped into a dense hypersphere and can be separated from other clusters. Soft projected clustering algorithms are driven by evaluation criteria and search strategies. Consequently, how to define the objective function and how to efficiently determine the optimal variable weighting are the two most important issues in soft projected clustering.

In this paper, we propose a particle swarm optimizer to obtain optimal variable weights in soft projected clustering of high-dimensional data. Good clustering quality is very dependent on a suitable objective function and an efficient search strategy. Our new soft projected clustering algorithm employs a special $k$-means objective weighting function, which takes the sum of the within-cluster distances of each cluster along relevant dimensions preferentially to irrelevant ones. If a variable is considered insignificant and assigned a small weight, the corresponding distance along this dimension will thus tend to be zero because the weights are handled exponentially. As a result, the function is more sensitive to changes in variable weights. In order to precisely measure the contribution of each dimension to each cluster, we assign a weight to each dimension in each cluster, following Domeniconi et al. (2007), Jing et al. (2007). 
The new algorithm also utilizes a normalized representation of variable weights in the objective function and transforms the constrained search space for the variable weighting problem in soft projected clustering into a redundant closed space, which greatly facilitates the search process. Instead of employing local search strategies, the new algorithm makes full use of a particle swarm optimizer to minimize the given objective function. It is simple to implement and the results are much less sensitive to the initial cluster centroids than those of other soft projected clustering algorithms. Experimental results on both synthetic datasets from a data generator and real datasets from the UCI database show that it can greatly improve cluster quality.

The rest of the paper is organized as follows. Section 2 reviews some previous related work on the variable weighting problem in soft projected clustering, explains the rationale behind using PSO for this problem and introduces the principle of PSO. Section 3 utilizes a particle swarm optimizer to assign the optimal variable weight vector for each cluster in soft projected clustering. Simulations on synthetic data are given in Section 4, which contains the description of synthetic datasets with different characteristics, the experimental setting for the PSO, and experimental results. In Section 5, experimental results on three real datasets are presented. An application of our new soft projected clustering algorithm to handle the problem of text clustering is given in Section 6. Section 7 draws conclusions and gives directions for future work.

\section{Previous work}

\subsection{Soft projected clustering}

Assuming that the number of clusters $k$ is given and that all of the variables for the datasets are comparable, soft projected clustering algorithms (Domeniconi et al. 2007; Huang et al. 2005; Jing et al. 2007) have been designed to discover clusters in the full dimensional space, by assigning a weight to each dimension for each cluster. The variable weighting problem is an important issue in data mining (Makarenkov and Legendre 2001). Usually, variables that correlate strongly with a cluster obtain large weights, which mean that these variables contribute strongly to the identification of data objects in the cluster. Irrelevant variables in a cluster obtain small weights. Thus, the computation of the membership of a data object in a cluster depends on the variable weights and the cluster centroids. The most relevant variables for each cluster can often be identified by the weights after clustering.

The performance of soft projected clustering largely depends on the use of a suitable objective function and an efficient search strategy. The objective function determines the quality of the partitioning, and the search strategy has an impact on whether the optimum of the objective function can be reached. Recently, several soft projected clustering algorithms have been proposed to perform the task of projected clustering and to select relevant variables for clustering.

Domeniconi et al. proposed the LAC algorithm, which determines the clusters by minimizing the following objective function (Domeniconi et al. 2007):

$$
\begin{aligned}
& F(w)=\sum_{l=1}^{k} \sum_{j=1}^{m}\left(w_{l, j} \cdot X_{l, j}+h \cdot w_{l, j} \cdot \log w_{l, j}\right), \quad X_{l, j}=\frac{\sum_{i=1}^{n} u_{i, l} \cdot\left(x_{i, j}-z_{l, j}\right)^{2}}{\sum_{i=1}^{n} u_{i, l}}, \\
& \text { s.t. }\left\{\begin{array}{l}
\sum_{j=1}^{m} w_{l, j}=1, \quad 0 \leq w_{l, j} \leq 1,1 \leq l \leq k, \\
\sum_{l=1}^{k} u_{i, l}=1, \quad u_{i, l} \in\{0,1\}, 1 \leq i \leq n,
\end{array}\right.
\end{aligned}
$$


where $k, n$ and $m$ are the number of clusters, the number of data objects and the number of dimensions, respectively. Throughout this paper, we will adopt the same notation. $u_{i, l}$ is the membership of data object $i$ in cluster $l . x_{i, j}$ is the value of data object $i$ on dimension $j$ and $z_{l, j}$ is the centroid of cluster $l$ on dimension $j . d$ is the distance function measuring the dissimilarity between two data objects. $X_{l, j}$ represents the squared variance of cluster $l$ along dimension $j . w_{l, j}$ is a weight assigned to cluster $l$ on dimension $j . U, Z$, and $W$ represent the cluster membership matrix of data objects, the cluster centroids matrix, and the dimensional weights matrix, respectively. The LAC algorithm is summarized as follows:

\section{Input:}

- Select $k$ well-scattered data objects as $k$ initial centroids.

- Set $w_{l, j}=1 / m$ for each dimension in each cluster.

\section{Repeat:}

- Update the cluster memberships of data objects $U$ by (3).

- Update the cluster centroids $Z$ by (4).

- Update the dimension weights $W$ by (5).

Until: (the objective function obtains its local minimum value, or the number of function evaluations reaches the maximum threshold).

The LAC algorithm finds a solution that is a local minimum of the above objective function. In the LAC algorithm, $U$ and $Z$ are updated in the same way as in the $k$-means algorithm.

$$
\begin{gathered}
\left\{\begin{array}{l}
u_{l, i}=1, \quad \text { if } \sum_{j=1}^{m} w_{l, j} \cdot\left(x_{i, j}-z_{l, j}\right)^{2} \leq \sum_{j=1}^{m} w_{t, j} \cdot\left(x_{i, j}-z_{t, j}\right)^{2} \text { for } 1 \leq t \leq k, \\
u_{l, i}=0, \quad \text { for } t \neq l,
\end{array}\right. \\
z_{l, j}=\frac{\sum_{i=1}^{n} u_{l, i} \cdot x_{i, j}}{\sum_{i=1}^{n} u_{l, i}}, \quad \text { for } 1 \leq l \leq k \text { and } 1 \leq j \leq m .
\end{gathered}
$$

$W$ is updated according to the following formula:

$$
w_{l, j}=\frac{\exp \left(-X_{l, j} / h\right)}{\sum_{t=1}^{m} \exp \left(-X_{l, t} / h\right)},
$$

where $h$ is a parameter that is used to control the deviation of the distribution of weight values from the uniform distribution through controlling the influence of $X$ on $w_{l, j}$.

In LAC 2007, the authors employed a new objective function and a weight updating schema. Their work is similar to the work described in EWKM (2007). In LAC 2007, the authors also proposed a method based on well-scattered data to initialize the cluster centroids and an ensemble approach to overcome the difficulty of tuning the parameter $h$. However, their initialization needs to calculate the distance between high-dimensional data in the whole dimension space, whereas the very hypothesis of this work is that this distance is not reliable. It is not clear yet whether LAC is significantly improved by the new initialization schema.

Huang et al. (2005) proposed another objective function and derived an algorithm called the $W-k$-means algorithm.

$$
F(w)=\sum_{l=1}^{k} \sum_{i=1}^{n} \sum_{j=1}^{m} u_{l, i} \cdot w_{j}^{\beta} \cdot d\left(x_{i, j}, z_{l, j}\right),
$$




$$
\text { s.t. } \begin{cases}\sum_{j=1}^{m} w_{j}=1, & 0 \leq w_{j} \leq 1,1 \leq l \leq k, \\ \sum_{l=1}^{k} u_{i, l}=1, & u_{i, l} \in\{0,1\}, 1 \leq i \leq n .\end{cases}
$$

Here, $\beta$ is a parameter greater than 1 . The objective function is designed to measure the sum of the within-cluster distances along variable subspaces rather than over the entire variable (dimension) space. The variable weights in the $W-k$-means need to meet the equality constraints, which are similar to those of (2) in LAC. The formula for updating the weights is different and is written as follows:

$$
w_{j}= \begin{cases}0, & \text { if } D_{j}=0, \\ \frac{1}{\sum_{t=1}^{m}\left[\frac{D_{j}}{D_{t}}\right]^{1 /(\beta-1)}}, & D_{j}=\sum_{l=1}^{k} \sum_{i=1}^{n} u_{i, l} \cdot d\left(x_{i, j}, z_{l, j}\right),\end{cases}
$$

where the weight $w_{j}$ for the $j$-th dimension is inversely proportional to the sum of all the within-cluster distances along dimension $j$. A large weight value corresponds to a small sum of within-cluster distances in a dimension, indicating that the dimension is more important in forming the clusters.

The $W$ - $k$-means algorithm is a direct extension of the $k$-means algorithm. The $W-k-$ means assigns a weight to each variable and seeks to minimize the sum of all the withincluster distances in the same subset of variables. Furthermore, updating of variable weights is dependent on the value of the parameter $\beta$. In addition, the $W$ - $k$-means algorithm does not utilize an efficient search strategy. Consequently, it has difficulty to correctly discover clusters embedded in different subsets of variables. Because it assigns a unique weight to each variable for all the clusters, the $W$ - $k$-means algorithm is not appropriate in the most cases of high-dimensional data where each cluster has its own relevant subset of variables.

To improve the $W-k$-means algorithm, Jing et al. (2007) proposed the entropy weighting $k$-means algorithm (EWKM), which assigns one weight to each variable for each cluster and employs a similar iterative process to that used by the $W-k$-means algorithm. The objective function in the EWKM algorithm takes both the within-cluster dispersion and the weight entropy into consideration. The function is described as follows:

$$
\begin{aligned}
& F(w)=\sum_{l=1}^{k}\left[\sum_{i=1}^{n} \sum_{j=1}^{m} u_{i, l} \cdot w_{l, j} \cdot\left(x_{i, j}-z_{l, j}\right)^{2}+\gamma \cdot \sum_{j=1}^{m} w_{l, j} \cdot \log w_{l, j}\right], \\
& \text { s.t. }\left\{\begin{array}{l}
\sum_{j=1}^{m} w_{l, j}=1, \quad 0 \leq w_{l, j} \leq 1,1 \leq l \leq k, \\
\sum_{l=1}^{k} u_{i, l}=1, \quad u_{i, l} \in\{0,1\}, 1 \leq i \leq n .
\end{array}\right.
\end{aligned}
$$

The constraints in (10) of the above function are identical to those in the LAC algorithm. The formula for updating the weights is written as follows:

$$
w_{l, j}=\frac{\exp \left(-D_{l, j} / \gamma\right)}{\sum_{t=1}^{m} \exp \left(-D_{l, t} / \gamma\right)}, \quad D_{l, j}=\sum_{i=1}^{n} u_{i, l} \cdot\left(x_{i, j}-z_{l, j}\right)^{2} .
$$

The objective (9) depends heavily on the value of parameter $\gamma$. If $\gamma$ is too small, the entropy section has little effect on the function. On the other hand, if $\gamma$ is too big, the entropy section could have too strong an effect on the function. The value of $\gamma$ is empirically determined and application-specific. 
The computational complexity of LAC, the $W-k$-means and EWKM is $O(m n k T)$, where $T$ is the total number of iterations and $m, n, k$ are the number of dimensions, the number of data objects and the number of clusters, respectively. Their computational complexity is low and increases linearly as the number of dimensions, the number of data objects or the number of clusters increases. Thus, these three principal algorithms for soft projected clustering scale well with the data size. They can converge to a local minimal solution after a finite number of iterations. However, since LAC, the $W$ - $k$-means and EWKM all stem from the $k$-means algorithm, they share some common problems. First, the constrained objective functions they provide have their drawbacks, which have been described above. Second, the cluster quality they yield is highly sensitive to the initial cluster centroids (see experiments). All the three algorithms employ local search strategies to optimize their objective functions with constraints. The local search strategies provide good convergence speed to the iterative process but do not guarantee good clustering quality because of local optimality.

\subsection{Rationale for using PSO for the variable weighting problem}

Basically, the variable weighting problem in soft projected clustering is a continuous nonlinear optimization problem with many equality constraints. It is a difficult problem, and has been researched for decades in the optimization field. Computational intelligence-based techniques, such as the genetic algorithm (GA), particle swarm optimization (PSO) and ant colony optimization (ACO), have been widely used to solve the problem.

GA is a heuristic search technique used in computing to find exact or approximate solutions to optimization problems. Genetic algorithms are a particular class of evolutionary computation that use techniques inspired by evolutionary biology, such as inheritance, mutation, selection, and crossover. In GA, the individuals of the population are usually represented by candidate solutions to an optimization problem. The population starts from randomly generated individuals and evolves towards better solutions over generations. In each generation, the fitness of every individual is evaluated; multiple individuals are selected from the current population based on their fitness, and recombined and mutated to form a new population. The population iterates until the algorithm terminates, by which time a satisfactory solution may be reached.

ACO is a swarm intelligence technique introduced by Marco Dorigo in 1992. It is a probabilistic technique for solving computational problems which can be reduced to finding good paths through graphs. In ACO, ants initially wander randomly and, upon finding food, return to the colony while laying down pheromone trails. Other ants follow the trail, returning and reinforcing it if they find food. However, the pheromone trail starts to evaporate over time, lest the paths found by the first ants should tend to be excessively attractive to the following ones. A short path gets marched over comparatively faster, and thus the pheromone density remains high as it is laid on the path as fast as it can evaporate. Thus, when one ant finds a good (i.e., short) path from the colony to a food source, other ants are more likely to follow that path, and positive feedback eventually results in all the ants following a single path.

PSO is another swarm intelligence technique, first developed by James Kennedy and Russell C. Eberhart in 1995. It was inspired by the social behavior of bird flocking and fish schooling. PSO simulates social behavior. In PSO, each solution to a given problem is regarded as a particle in the search space. Each particle has a position (usually a solution to the problem) and a velocity. The positions are evaluated by a user-defined fitness function. At each iteration, the velocities of the particles are adjusted according to the historical best positions of the population. The particles fly towards better regions through their own effort and in cooperation with other particles. Naturally, the population evolves to an optimal or near-optimal solution. 
PSO can generate a high-quality solution within an acceptable calculation time and a stable convergence characteristic in many problems where most other methods fail to converge. It can thus be effectively applied to various optimization problems. A number of papers have been published in the past few years that focus on the applications of PSO, such as neural network training (Bergh and Engelbecht 2000), power and voltage control (Yoshida et al. 2000), task assignment (Salman et al. 2003), single machine total weighted tardiness problems (Tasgetiren et al. 2004) and automated drilling (Onwubolu and Clerc 2004). Moreover, PSO has some advantages over other similar computational intelligence-based techniques such as GA and ACO for solving the variable weighting problem for high-dimensional clustering. For instance:

(1) PSO is easier to implement and more computationally efficient than GA and ACO. There are fewer parameters to adjust. PSO only deals with two simple arithmetic operations (addition and multiplication), while GA needs to handle more complex selection and mutation operators.

(2) In PSO, every particle remembers its own historic best position, while every ant in ACO needs to track down a series of its own previous positions and individuals in GA have no memory at all. Therefore, a particle in PSO requires less time to calculate its fitness value than an ant in ACO due to the simpler memory mechanism, while sharing the strong memory capability of the ant at the same time. PSO has a more effective memory capability than GA.

(3) PSO is more efficient in preserving population diversity to avoid the premature convergence problem. In PSO, all the particles improve themselves using their own previous best positions and are improved by other particles' previous best position by cooperating with each other. In GA, there is "survival of the fittest". The worst individuals are discarded and only the good ones survive. ACO easily loses population diversity because ants are attracted by the largest pheromone trail and there is no direct cooperation among ants.

(4) PSO has been found to be robust, especially in solving continuous nonlinear optimization problems, while GA and ACO are good choices for constrained discrete optimization problems. ACO has an advantage over other evolutionary approaches when the graph may change dynamically, and can be run continuously and adapt to changes in real time.

Since the PSO method is an excellent optimization methodology for solving complex parameter estimation problems, we have developed a PSO-based algorithm for computing the optimal variable weights in soft projected clustering.

\subsection{PSO}

In PSO, each solution is regarded as a particle in the search space. Each particle has a position (usually a solution to the problem) and a velocity. The particles fly through the search space through their own effort and in cooperation with other particles.

The velocity and position updates of the $i$ th particle are as follows:

$$
\begin{aligned}
& V_{i}(t+1)=\lambda \cdot V_{i}(t)+c 1 \cdot r 1 \cdot\left(\text { pBest }_{i}-X_{i}(t)\right)+c 2 \cdot r 2 \cdot\left(g \text { Best }-X_{i}(t)\right), \\
& X_{i}(t+1)=V_{i}(t+1)+X_{i}(t),
\end{aligned}
$$


where $X_{i}$ is the position of the $i$ th particle, $V_{i}$ presents its velocity and $p B e s t_{i}$ is its personal best position yielding the best function value for it. $g B e s t$ is the global best position discovered by the whole swarm. $\lambda$ is the inertia weight used to balance the global and local search capabilities. $c 1$ and $c 2$ are the acceleration constants, which represent the weights pulling each particle toward the pbest and gbest positions, respectively. $r 1$ and $r 2$ are two random numbers uniformly distributed in the range $[0,1]$.

The original PSO algorithm was later modified by the researchers in order to improve its performance. Comprehensive Learning Particle Swarm Optimizer (CLPSO), which is one of the well-known modifications of PSO, was proposed by Liang (2006) to efficiently optimize multimodal functions. The following velocity update was employed in CLPSO:

$$
V_{i}(t+1)=\lambda \cdot V_{i}(t)+c 1 \cdot r 1 \cdot\left(\text { CpBest }_{i}-X_{i}\right)
$$

where Cpbest $_{i}$ is a comprehensive learning result from the personal best positions of some particles. CLPSO achieves good diversity and effectively solves the premature convergence problem in PSO. The CLPSO algorithm for a minimum optimization problem is summarized as follows:

\section{Initialization:}

- Randomly initialize the position and velocity swarms, $X$ and $V$.

- Evaluate the fitness of $X$ by the objective function.

- Record the personal best position swarm pBest and the global best position gBest.

\section{Repeat:}

- Produce CpBest from pBest for each particle.

- Update $V$ and $X$ by (13) and (12), respectively.

- Update pBest and gBest.

Until: (the objective function reaches a global minimum value, or the number of function evaluations reaches the maximum threshold).

\section{PSO for variable weighting}

In this section, we proposed a PSO-based algorithm, called PSOVW, to solve the variable weighting problem in soft projected clustering of high-dimensional data. We begin by showing how the problem of minimization of a special $k$-means function with equality constraints can be transformed into a nonlinear optimization problem with bound constraints, using a normalized representation of variable weights in the objective function, and how a particle swarm optimizer can be used to control the search progress. We then give a detailed description of our PSOVW algorithm, which minimizes the transformed problem. Finally, we discuss the computational complexity of the proposed algorithm.

\subsection{PSO for variable weighting (PSOVW)}

Our particle swarm optimizer for the variable weighting problem in soft projected clustering, termed PSOVW, performs on two main swarms of variable weights, the position swarm $W$ and the personal best position swarm of variable weights pBest. The position swarm is the 
foundation of the search in the variable weights space, so the evolution of the position swarm pays more attention to global search, while the personal best position swarm tracks the fittest positions, in order to accelerate the convergence. In addition, it facilitates cooperation among the previous best positions in order to maintain the swarm diversity.

\section{The objective function}

We minimize the following objective function in PSOVW:

$$
\begin{aligned}
& F(W)=\sum_{l=1}^{k} \sum_{i=1}^{n} \sum_{j=1}^{m} u_{l, i} \cdot\left(\frac{w_{l, j}}{\sum_{j=1}^{m} w_{l, j}}\right)^{\beta} \cdot d\left(x_{i, j}, z_{l, j}\right), \\
& \text { s.t. } \quad\left\{\begin{array}{l}
0 \leq w_{l, j} \leq 1, \\
\sum_{l=1}^{k} u_{i, l}=1, \quad u_{i, l} \in\{0,1\}, 1 \leq i \leq n .
\end{array}\right.
\end{aligned}
$$

Actually, the objective function in (14) is a generalization of some existing objective functions. If $\beta=0,(14)$ is similar to the objective function used in the $k$-means. They differ solely in the representation of variable weights and the constraints. If $\beta=1$, (14) is also similar to the first section of the objective function in EWKM, differing only in the representation of variable weights and the constraints. If $w_{l, j}=w_{j}, \forall l,(14)$ is similar to the objective function in the $W-k$-means, which assigns a single variable weight vector, substituting different vectors for clusters. Again, the difference is in the representation of variable weights and the constraints.

In PSOVW, $\beta$ is a user-defined parameter. PSOVW works with all non-negative values of $\beta$. In practice, we suggest setting $\beta$ to a large value (empirically around 8 ). A large value of $\beta$ makes the objective function more sensitive to changes in weight values. It tends to magnify the influence of those variables with large weights on the within-cluster variance, allowing them to play a strong role in discriminating between relevant and irrelevant variables (dimensions). On the other hand, in contrast to LAC and EWKM algorithms in which the variable weight matrix $W$ is part of the solution to the objective function and must meet the equality constraints, a normalized matrix $W$ is employed in the objective function in PSOVW. Although the normalized representation in the constrained objective function (14) is redundant, the constraints can be loosened without affecting the final solution. As a result, the initialization and search processes only need to deal with bound constraints instead of many more complicated equality constraints. PSOVW includes three parts.

\section{Initialization}

In the PSOVW algorithm, we initialize three swarms.

- The position swarm $W$ of variable weights are set to random numbers uniformly distributed in a certain range $R$.

- The velocity swarm $V$ of variable weights are set to random numbers uniformly distributed in the range $[-\max v, \max v]$. Here, $\max v$ means the maximum flying velocity of particles and $\max v=0.25 * R$.

- The swarm $Z$ of cluster centroids are $k$ different data objects randomly chosen out of all the data objects.

In all three swarms, an individual is the $k * m$ matrix. Actually, only the velocity swarm

$V$ is an extra swarm not included in $k$-means-type soft projected clustering algorithms. 


\section{Update of cluster centroids and partitioning of data objects}

Given the variable weight matrix and the cluster centroids, the cluster membership of each data object is calculated by the following formula.

$$
\left\{\begin{aligned}
u_{l, i}=1, & \text { if } \sum_{j=1}^{m}\left(\frac{w_{l, j}}{\sum_{j=1}^{m} w_{l, j}}\right)^{\beta} \cdot d\left(z_{l, j}, x_{i, j}\right) \leq \sum_{j=1}^{m}\left(\frac{w_{q, j}}{\sum_{j=1}^{m} w_{q, j}}\right)^{\beta} \cdot d\left(z_{t, j}, x_{i, j}\right) \\
& \text { for } 1 \leq q \leq k, \\
u_{l, i}=0, & \text { for } q \neq l
\end{aligned}\right.
$$

Once the cluster membership is obtained, the cluster centroids are updated by

$$
z_{l, j}=\left(\sum_{i=1}^{n} u_{l, i} \cdot x_{i, j}\right) /\left(\sum_{i=1}^{n} u_{l, i}\right), \quad \text { for } 1 \leq l \leq k \text { and } 1 \leq j \leq m .
$$

In our implementation, if an empty cluster results from the membership update by formula (16), we randomly select a data object out of the dataset to reinitialize the centroid of the cluster.

\section{Crossover learning}

Given a value for $\beta$, two extra swarms are kept to guide all the particles' movement in the search space. One is the personal best position swarm of variable weights $p B e s t$, which keeps the best position of the weight matrix $W$; i.e., pBest will be replaced by $W$ if $F(W)<$ $F$ (pBest). The other is the crossover best position swarm of variable weights $C p B e s t$, which guides particles to move towards better regions (Liang et al. 2006). CpBest is obtained by a crossover operation between its own $p B e s t$ and one of the other best personal positions in the swarm, represented here by $s \_p B e s t$.

The tournament mechanism is employed to select $s \_p B e s t$, with the consideration that a particle learns from a good exemplar. First, two individuals, pBest 1 and pBest2, are randomly selected. Then their objective function values are compared: $s \_p B e s t=p B e s t 1$ if $F(p B e s t)<F(p B e s t 2), s_{\_} p B e s t=p B e s t 2$ otherwise. The crossover is done as follows. Each element at a position of $C p B e s t$ is assigned the value either from $s \_p B e s t$ or from $p B e s t$ at the corresponding position. This assignment is made randomly according to a user-defined probability value $P_{c}$ (see the section Synthetic Data Simulations for further discussion). If, despite the random assignment process, all the elements of $C p B e s t$ take values from its own $p B e s t$, one element of CpBest will be randomly selected and its value will be replaced by the value of the corresponding position from $s_{\_} p B e s t$.

The PSOVW algorithm can be summarized as follows:

\section{Initialization:}

- Randomly initialize the position swarm $W$ in the range of $[0,1]$ and the velocity swarm $V$ in the range of $[-m v, m v]$, where $m v=0.25 *(1-0)$. Randomly select a set of $k$ data objects out of the dataset as a set of $k$ cluster centroids in the cluster centroid swarm.

- Partition the data objects by the formula (16).

- For each position $W$,

If $W$ lies in the range [0,1],

Evaluate the fitness function value $F(W)$ of $W$ by the formula (14).

Record the swarm pBest and the best position gBest. 
Otherwise,

$W$ is neglected.

End

\section{Repeat:}

- For each position $W$,

Produce CpBest from the swarm $p$ Bests.

Update $V$ and $W$ by (13) and (12), respectively.

If $W$ lies in the range $[0,1]$,

Evaluate its fitness by (14).

Update the position's pBest and gBest.

Otherwise,

$W$ is neglected.

End.

Until: (the objective function reaches a global minimum value, or the number of function evaluations reaches the maximum threshold).

\subsection{Computational complexity}

The computational complexity can be analyzed as follows. In the PSOVW algorithm, the $C p B e s t$, position and velocity of a particle are $k * m$ matrices. In the main loop procedure, after initialization of the position and velocity, generating $C p B e s t$ and updating position and velocity need $O(m k)$ operations for each particle. Given the weight matrix $W$ and the cluster centroids matrix $Z$, the complexity for partitioning the data objects is $O(m n k)$ for each particle. The complexity for updating cluster centers is $O(m k)$ for each particle. Assuming that the PSOVW algorithm needs $T$ iterations to converge, the total computational complexity of the PSOVW algorithm is $O(\operatorname{smnkT})$. The swarm size $s$ is usually a constant set by the user. So, the computational complexity still increases linearly as the number of dimensions, the number of objects or the number of clusters increases.

\section{Synthetic data simulations}

A comprehensive performance study was conducted to evaluate the performance of PSOVW on high-dimensional synthetic datasets as well as on real datasets. Three of the most widely used soft projected clustering algorithms, LAC (Domeniconi et al. 2007), the $W-k$-means (Huang et al. 2005) and EWKM (Jing et al. 2007), were chosen for comparison, as well as the basic $k$-means. ${ }^{1}$ The goal of the experiments was to assess the accuracy and efficiency of PSOVW. We compared the clustering accuracy of the five algorithms, examined the variance of the cluster results and measured the running time spent by these five algorithms. These experiments provide information on how well and how fast each algorithm is able to retrieve known clusters in subspaces of very high-dimensional datasets, under various conditions of cluster overlapping, and how sensitive each algorithm is to the initial centroids. In this section, we describe the synthetic datasets and the results.

\footnotetext{
${ }^{1}$ The $k$-means is included in order to show that projected clustering algorithms are necessary on highdimensional data sets.
} 


\subsection{Synthetic datasets}

We generated high-dimensional datasets with clusters embedded in different subspaces using a data generator, derived from the generation algorithm in Jing (2007) (see Appendix for the algorithm). In order to better measure the difficulties of the generated datasets, we explicitly controlled three parameters in the generator. Here we assume that the (sub)space of a cluster consists of its relevant dimensions.

Parameter 1 The subspace ratio, $\varepsilon$, of a cluster, as defined in Jing et al. (2007), is the average ratio of the dimension of the subspace to that of the whole space. $\varepsilon \in[0,1]$. It is set to 0.375 in our experiments.

Parameter 2 The (relevant) dimension overlap ratio, $\rho$, of a cluster, also defined in Jing et al. (2007), is the ratio of the dimension of the overlapping subspace, which also belongs to another cluster, to the dimension of its own subspace. $\rho \in[0,1]$. For example, the subspace of cluster $A$ is $\{1,6,7,11,16,19,20,21\}$, while that of cluster $B$ in the same dataset is $\{1,3,6,7,8,11,12,15,16\}$. The dimension size of the subspace of cluster $A$ is 8 . The overlapping subspace of $A$ and $B$ is $\{1,6,7,11,16\}$ and its size is 5. The dimension overlap ratio of cluster $A$ with respect to cluster $B$ is $\rho=0.625$. For a given $\rho$, each generated cluster is guaranteed to have a dimension overlap ratio $\rho$ with at least one other cluster. In other words, the dimension overlap ratio of a generated cluster w.r.t. any other generated cluster could be either greater or smaller than $\rho$. Nevertheless, the dimension overlap ratio is still a good indicator of the overlapping of relevant dimensions between clusters in a dataset.

Parameter 3 The data overlap ratio, $\alpha$, is a special case of the overlapping rate concept that we previously proposed in Aitnouri et al. (2000) and Bouguessa et al. (2006). In Aitnouri et al. (2000) and Bouguessa et al. (2006), the overlapping rate between two Gaussian clusters was defined as the ratio of the minimum of the mixture $p d f$ (probability distribution function) on the ridge curve linking the centers of two clusters to the height of the lower peak of the $p d f$. It has been shown that this overlapping rate is a better indicator of the separability between two clusters than the conventional concept of overlapping rate based on "Bayesian classification error". In the one-dimensional case, if the variance of the two clusters is fixed, then the overlap rate is completely determined by the distance between the two centroids. In our data generation procedure, the data overlap ratio $\alpha$ is such a parameter that controls the distance between the centroid of the cluster currently being generated and the centroid of the cluster generated in the previous step on each of the overlapped relevant dimensions.

Three groups of synthetic datasets were generated. They differ in the number of dimensions, which are 100, 1000 and 2000. In these synthetic datasets, different clusters have their own relevant dimensions, which can overlap. The data values are normally distributed on each relevant dimension of each cluster, the corresponding mean is specified in the range $[0,100]$ and the corresponding variance is set to 1 . Random positive numbers are on the irrelevant dimensions and random values are uniformly distributed in the range $[0,10]$. We generated a total of 36 synthetic datasets with different values of $\rho$ and different values of $\alpha$, with $\rho$ taking a value from the set $\{0.2,0.5,0.8\}$ and $\alpha$ taking a value from another set $\{0.2,0.5,1,2\}$. In general, the larger the value of $\rho$ and the smaller the value of $\alpha$, the more complicated (in terms of dimension overlapping and data overlapping in common relevant dimensions) the synthetic datasets are. The subspace ratio $\varepsilon$ is set to 0.375 . For each group 
of data, there are 12 datasets, each of which has 500 data objects, 10 clusters and 50 data objects in a cluster.

The Matlab coding for generating the three groups of datasets and the $\mathrm{C}++$ coding for the implementation of these five clustering algorithms can be obtained from the authors.

\subsection{Parameter settings for algorithms}

The Euclidean distance is used to measure the dissimilarity between two data objects for synthetic and real datasets. Here, we set $\beta=8$. The maximum number of function evaluations is set to 500 over all synthetic datasets in PSOVW and 100 in the other four algorithms. Given an objective function, LAC, the $W-k$-means and EWKM quickly converge to local optima after a few iterations, while PSOVW needs more iterations to get to its best optima. There are four additional parameters in PSOVW that need to be specified. These are the swarm size $s$, the inertia weight $\lambda$, the acceleration constant $c 1$ and the learning probability $P_{c}$. Actually, these four extra parameters have been fully studied in the particle swarm optimization field and we set the parameters in PSOVW to the values used in Liang et al. (2006). $s$ is set to $10 . \alpha$ is linearly decreased from 0.9 to 0.7 during the iterative procedure, and $c 1$ is set to 1.49445 . In order to obtain good population diversity, particles in PSOVW are required to have different exploration and exploitation ability. So, $P_{c}$ is empirically set to $P_{c}(i)=0.5 * \frac{e^{f(i)}-e^{f(1)}}{e^{f(s)}-e^{f(1)}}$, where $f(i)=5 * \frac{i}{s-1}$ for each particle. The values of the learning probability $P_{c}$ in PSOVW range from 0.05 to 0.5 , as for CLPSO (Liang et al. 2006). The parameter $\gamma$ in EWKM is set to 1 . We also set the parameter $\beta$ in the $W$ - $k$-means to 8 as the authors did in their paper (Huang et al. 2005).

\subsection{Results for synthetic data}

To test the clustering performance of PSOVW, we first present the average clustering accuracy of the five algorithms ( $k$-means, LAC, $W$ - $k$-means, EWKM and PSOVW). Then we employ the PSO method with different functions on several selected synthetic datasets and report the clustering results yielded by them. This provides further evidence for the superior effectiveness of PSOVW in clustering high-dimensional data. Finally, we examine the average clustering accuracy, the variance of the clustering results and the running time of the five algorithms on synthetic datasets, and make an extensive comparison between them. The clustering accuracy is calculated as follows:

$$
\text { Clustering Accuracy }=\sum_{l=1}^{k} n_{l} / n,
$$

where $n_{l}$ is the number of data objects that are correctly identified in the genuine cluster $l$ and $n$ is the number of data objects in the dataset. The clustering accuracy is the percentage of the data objects that are correctly recovered by an algorithm.

In order to compare the clustering performance of PSOVW with those of the $k$-means, LAC, ${ }^{2}$ the $W$ - $k$-means and EWKM, we ran these five algorithms on datasets from each group. Since PSOVW was randomly initialized and the other algorithms are sensitive to the

\footnotetext{
${ }^{2}$ Since it is difficult to simulate the function combining different weighting vectors in the latest LAC algorithm (Domeniconi et al. 2007), we tested its performance with the values from 1 to 11 for the parameter $1 / h$, as the authors did in Domeniconi et al. (2007), and report the best clustering accuracy on each trial.
} 
Table 1 Average clustering accuracy of PSOVW, $k$-means, $W$ - $k$-means, EWKM and LAC over 20 trials on each dataset with 100 dimensions

\begin{tabular}{llllll}
\hline$\rho \backslash \alpha$ & 0.2 & 0.5 & 1 & 2 & Algorithms \\
\hline 0.2 & 82.76 & 91.16 & 92.56 & 91.68 & PSOVW \\
& 69.68 & 65.00 & 68.16 & 61.20 & EWKM \\
& 68.44 & 72.00 & 69.68 & 78.24 & $W$ - $k$-means \\
& 68.12 & 64.48 & 69.04 & 65.08 & LAC \\
& 52.88 & 42.04 & 50.40 & 46.60 & $k$-means \\
0.5 & 83.12 & 85.96 & 86.40 & 89.40 & PSOVW \\
& 66.28 & 61.24 & 59.56 & 68.88 & EWKM \\
& 62.84 & 70.24 & 75.49 & 76.82 & LAC \\
& 69.48 & 66.88 & 63.16 & 60.24 & $k$-means \\
& 39.56 & 43.36 & 36.55 & 39.24 & PSOVW \\
& 80.24 & 83.80 & 83.68 & 83.92 & EWKM \\
& 65.76 & 71.00 & 61.80 & 56.64 & $W$ - $k$-means \\
& 72.24 & 76.96 & 77.64 & 83.40 & LAC \\
& 61.56 & 58.24 & 69.76 & 62.80 & $k$-means \\
\hline
\end{tabular}

Table 2 Average clustering accuracy of PSOVW, $k$-means, $W$ - $k$-means, EWKM and LAC over 20 trials on each dataset with 1000 dimensions

\begin{tabular}{llllll}
\hline$\rho \backslash \alpha$ & 0.2 & 0.5 & 1 & 2 & Algorithms \\
\hline 0.2 & 84.32 & 92.36 & 90.60 & 91.16 & PSOVW \\
& 55.12 & 63.72 & 62.52 & 54.80 & EWKM \\
& 60.28 & 68.56 & 82.16 & 69.12 & $W$ - $k$-means \\
& 66.80 & 65.52 & 65.00 & 68.12 & LAC \\
& 30.56 & 36.88 & 23.16 & 36.68 & $k$-means \\
& 83.92 & 87.84 & 90.84 & 89.24 & PSOVW \\
& 66.68 & 64.80 & 72.96 & 57.28 & EWKM \\
& 72.68 & 67.48 & 74.08 & 76.44 & LAC \\
& 56.04 & 73.88 & 68.52 & 61.72 & $k$-means \\
& 28.32 & 34.56 & 25.20 & 35.48 & PSOVW \\
& 80.36 & 86.12 & 88.84 & 84.68 & EWKM \\
& 64.36 & 63.72 & 68.24 & 52.84 & $W$ - $k$-means \\
& 77.52 & 73.12 & 75.00 & 80.12 & LAC \\
& 57.20 & 60.92 & 58.12 & 28.80 & $k$-means \\
\hline
\end{tabular}

initial cluster centroids to a certain degree, we ran each algorithm, including PSOVW, for 20 trials on each dataset. The average clustering accuracy achieved by each algorithm is recorded in Tables 1, 2 and 3. 
Table 3 The average clustering accuracy of PSOVW, $k$-means, $W$ - $k$-means, EWKM and LAC over 20 trials on each dataset with 2000 dimensions

\begin{tabular}{llllll}
\hline$\rho \backslash \alpha$ & 0.2 & 0.5 & 1 & 2 & Algorithms \\
\hline 0.2 & 82.87 & 85.01 & 87.01 & 90.84 & PSOVW \\
& 58.40 & 66.56 & 61.44 & 60.82 & EWKM \\
& 69.01 & 65.64 & 70.15 & 64.76 & $W$ - $k$-means \\
& 61.81 & 62.36 & 60.64 & 69.70 & LAC \\
& 42.56 & 36.33 & 38.49 & 43.22 & $k$-means \\
0.5 & 82.53 & 86.47 & 86.72 & 87.76 & PSOVW \\
& 68.09 & 65.84 & 64.64 & 71.22 & EWKM \\
& 67.84 & 73.80 & 68.95 & 68.04 & LAC \\
& 61.36 & 60.56 & 65.52 & 61.10 & $k$-means \\
& 34.15 & 25.15 & 27.56 & 31.68 & PSOVW \\
& 82.72 & 82.80 & 85.62 & 85.06 & EWKM \\
& 64.64 & 65.40 & 65.50 & 72.04 & $W$ - $k$-means \\
& 74.00 & 66.76 & 64.16 & 74.24 & $k$ LAC \\
& 65.52 & 67.46 & 27.41 & 24.82 & $k$-means \\
\hline
\end{tabular}

A number of observations can be made by analyzing the results in Tables 1, 2 and 3 . First, PSOVW seems to perform much better than the other algorithms tested on the three groups of generated datasets. In fact, it surpasses the $W$ - $k$-means, EWKM and LAC as well as the $k$-means on all 36 synthetic datasets. On the other hand, there is a clear trend in the performance of PSOVW that coincides with variations in the complexity of the datasets. Here, the performance of PSOVW increases with the data overlap ratio $\alpha$ and decreases with the dimension overlap ratio $\rho$ (with a few minor exceptions). This was to be expected, as the clusters are more separated with large $\alpha$ and share more common relevant dimensions with larger $\rho$.

Second, the $W-k$-means performs the next best on 27 of the 36 datasets. In general, the clustering performance of the $W-k$-means increases with $\alpha$ and $\rho$. From the tables, we can see that it achieves better accuracy than LAC, EWKM and the $k$-means on the datasets with large values of $\alpha$. There is also a clear trend for improved performance of the $W-k$-means with larger values of $\rho$. In fact, since it assigns one weight to each dimension for all the clusters, it is naturally more appropriate for datasets with clusters sharing a large number of relevant dimensions, while a large $\rho$ indicates that the subspaces for clusters tend to overlap greatly.

Third, from the experimental results reported in these three tables, we can see no clear trend in the performances of LAC and EWKM. Both algorithms assign one weight to each dimension of each cluster as in PSOVW, but perform poorly compared to PSOVW and the $W$ - $k$-means. In the initial papers (Huang et al. 2005; Jing et al. 2007) where these two algorithms were proposed, they are evaluated on datasets with relatively low dimensionality. The parameters, $\gamma$ and $h$, in the entropy section of the objective functions of EWKM and LAC are application-specific. It is possible that the poor performances of LAC and EWKM are due in part to our failure to find optimal values for these parameters. However, we also believe that the iterative schemes in these algorithms tend to converge quickly to low-quality local minima, and even more so in high-dimensional case, as with the challenging datasets 
used in this work. The fact that the obtained accuracy measure seems to be random with these two algorithms is a good indicator of the inefficiency of their search algorithms. This point is further validated in our experiments using PSO with different objective functions (see results reported below).

Finally, the clustering accuracy achieved by the $k$-means drops quickly as the dimension overlap $\rho$ increases. The $k$-means performs the worst on all the synthetic datasets. While this is not surprising, as it was not designed to deal with high-dimensional datasets, the $k$-means nevertheless exhibits a certain similarity to PSOVW in that it tends to perform well (or better) with large values of $\alpha$ and poorly (or worse) with large values of $\rho$. This partially confirms our assessment regarding the variations in the complexity of the data used.

In order to further investigate the reasons why PSOVW achieves better performance than other algorithms on generated high-dimensional datasets, we also implemented the PSO method with different objective functions presented in soft projected clustering algorithms, namely formula (1) in LAC, formula (6) in the $W-k$-means, formula (9) in EWKM and formula (14) in PSOVW. Usually, a search strategy is designed for a specific objective function with certain constraints. PSO is a heuristic global search strategy, so it is flexible and can be employed to optimize other objective functions. Since PSO was randomly initialized, the PSO method with each function was run for 20 trials on each of 9 selected datasets. $m$ represents the number of dimensions. The average clustering accuracy yielded by the PSO method with each function is recorded in Table 4. The results for PSOVW are taken from Tables 1, 2 and 3.

Table 4 gives the clustering results of the PSO method with different functions on 9 selected datasets. Obviously, the PSO method greatly improves the performances of LAC, the $W$ - $k$-means and EWKM. In our experiments, we also found that the high clustering accuracy yielded by the PSO method with different functions benefits greatly from the normalized representation of variable weights presented in our objective functions, although we also tried several existing techniques to initialize feasible particles. For a fair comparison, we implemented the same representation as in function (14) of PSOVW, where variable weights are only required to meet bound constraints, in the LAC, $W-k$-means and EWKM functions. From Table 4, we can see that on average, PSO with function (14) performs best on 6 of the 9 datasets, although PSO with other functions performs better than PSOVW in

Table 4 Average clustering accuracy of the PSO method with different functions over 20 trials on 9 selected datasets with different values of $\rho$ and $\alpha$, combined with different numbers of dimensions

\begin{tabular}{llrllll}
\hline$\rho$ & $\alpha$ & $m$ & $\begin{array}{l}\text { Function (14) } \\
\text { in PSOVW }\end{array}$ & $\begin{array}{l}\text { Function (9) } \\
\text { in EWKM }\end{array}$ & $\begin{array}{l}\text { Function (6) } \\
\text { in } W \text { - } k \text {-means }\end{array}$ & $\begin{array}{l}\text { Function (1) } \\
\text { in LAC }\end{array}$ \\
\hline 0.5 & 0.5 & 100 & $\mathbf{8 5 . 9 6}$ & 84.82 & 84.62 & 82.18 \\
& & 1000 & 87.84 & 90.06 & 84.06 & $\mathbf{9 0 . 1 8}$ \\
& & 2000 & 86.47 & $\mathbf{9 0 . 5 4}$ & 86.02 & 88.72 \\
0.5 & 1 & 100 & $\mathbf{8 6 . 4 0}$ & 84.50 & 81.78 & 85.50 \\
& & 1000 & $\mathbf{9 0 . 8 4}$ & 83.78 & 83.24 & 89.08 \\
& & 2000 & $\mathbf{8 6 . 7 2}$ & 85.76 & 85.24 & 85.74 \\
0.8 & 1 & 100 & 83.68 & 83.9 & $\mathbf{8 5 . 8 6}$ & 83.48 \\
& & 1000 & $\mathbf{8 8 . 8 4}$ & 83.24 & 86.04 & 84.52 \\
& & 2000 & 85.62 & 83.17 & $\mathbf{8 9 . 6 4}$ & 87.24 \\
\hline
\end{tabular}


some cases. The fact that function (6) is less efficient than functions (1), (9) and (14) is understandable because it uses one weight per dimension; nevertheless, it performs better than others on the datasets with large dimension overlap ratios.

The results reported in Tables 1, 2, 3 and 4 suggest that the good performance of PSOVW is due to the PSO method as well as to the normalized representation and the bound constraints on variable weights in our improved objective function.

In order to see how sensitive the clustering results of these five algorithms are to the initial cluster centroids, we also examined the variance of 20 clustering results yielded by each algorithm on each dataset. From Fig. 1, it can be readily seen that PSOVW yields the least variance in clustering accuracy on most of the datasets. The $k$-means achieves lower variance in clustering accuracy than PSOVW on some datasets with 10 clusters, because the clustering accuracy yielded by the $k$-means on these datasets is always very low. The other three soft projected clustering algorithms occasionally work well, achieving low variance in clustering accuracy on some datasets, but their variance is often high. What's more, it is difficult to tell for which kind of datasets they are less sensitive to the initial centroids. From the three graphs in Fig. 1, we conclude that PSOVW is far less sensitive to the initial centroids than the other four algorithms.

From Table 5, it is clear that the $k$-means runs faster than the other four soft projected clustering algorithms. Although LAC, EWKM and the $W$ - $k$-means all extend the $k$-means clustering process by adding an additional step in each iteration to compute variable weights for each cluster, LAC and EWKM are much faster algorithms than the $W$ - $k$-means on datasets. The $W$ - $k$-means required more time to identify clusters, because its objective function involves a much more complicated computation due to the high exponent. Based on the computational complexities of the various algorithms and the maximum number of function evaluations set in each algorithm, PSOVW was supposed to need $5 \sim 50$ times the running time the $W$ - $k$-means requires. In fact, it required only slightly around 4,2 and 2 times the running time that the $W$ - $k$-means spent on the datasets with 100, 1000 and 2000 dimensions, respectively. This is because the update of weights is simpler in PSOVW than in the other three soft projected clustering algorithms and particles are evaluated only if they are in the feasible bound space. We thus conclude that the much more complicated search strategy of PSOVW allows it to achieve the best clustering accuracy, at an acceptable cost in terms of running time.

Since PSOVW uses more resources than LAC, EWKM and the $W$ - $k$-means, to ensure a very fair comparison of PSOVW with these three soft projected clustering algorithms, we independently ran LAC, EWKM and the $W$ - $k$-means multiple times on each dataset and compared the best result out of the iterative trials with that of PSOVW. Here, we did not compare PSOVW with $k$-means, because $k$-means always works badly on these datasets. According to the average running time of each algorithm reported in Table 5, we ran PSOVW once on each of the nine datasets. On the datasets with 100, 1000 and 2000 dimensions, LAC and EWKM were run 6, 3 and 3 times respectively and $W$ - $k$-means was run 4, 2 and 2 times respectively. The experimental results in Table 6 still show that PSOVW surpasses the other three algorithms on each dataset except on two datasets. This is because particles in PSOVW do not work independently but cooperate with each other in order to move to better regions. The quality of the clustering is of prime importance and therefore the time taken to obtain it is at most secondary in many clustering applications. To sum up, the much more complicated search strategy allows PSOVW to achieve the best clustering accuracy, at a cost of longer running time, but the running time is acceptable. 


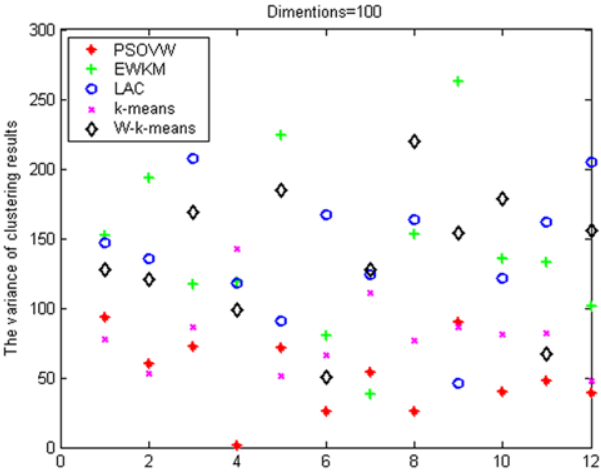

(a) Variance on datasets with 100 dimensions

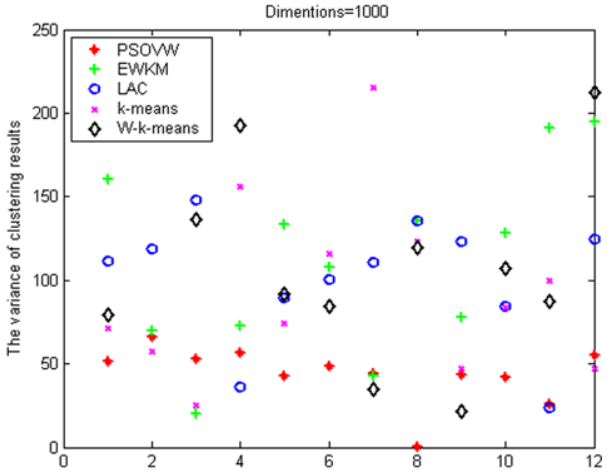

(b) Variance on datasets with 1000 dimensions

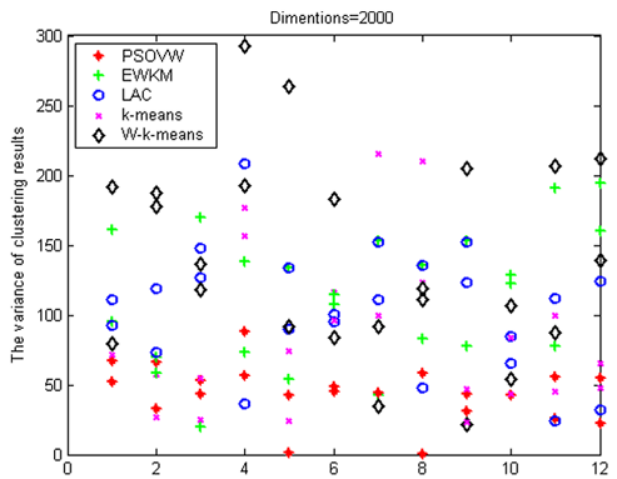

(c) Variance on datasets with 2000 dimensions

Fig. 1 Average clustering accuracy and corresponding variance of PSOVW, $k$-means, $W$ - $k$-means, EWKM and LAC on each dataset. The horizontal axis represents the 12 datasets in numerical order from 1 to 12 for each group. The vertical axis represents the variance of 20 clustering accuracies yielded by each algorithm on each dataset

Table 5 Average running time of PSOVW vs. $k$-means, LAC, $W$ - $k$-means, and EWKM on the datasets, with varying $m$. All times are in seconds

\begin{tabular}{llcccc}
\hline Datasets & \multicolumn{3}{l}{ Running Time (s) } & & \\
\cline { 2 - 5 }$m$ & $k$-means & EWKM & LAC & $W$ - $k$-means & PSOVW \\
\hline 100 & 0.42 & 6.64 & 7.28 & 11.95 & 38.86 \\
1000 & 3.83 & 66.17 & 72.357 & 119.09 & 186.572 \\
2000 & 6.67 & 131.9 & 144.51 & 238.21 & 310.85 \\
\hline
\end{tabular}

\section{Test on real data}

\subsection{Real-life datasets}

A comprehensive performance study was conducted to evaluate our method on real datasets. For the experiments described in this section, we chose three real datasets: the glass identification dataset (Evett and Spiehler 1987), the Wisconsin breast cancer dataset (Mangasarian 
Table 6 The comparison of the result of PSOVW once vs. the best result of LAC, EWKM and the $W-k$ means multiple independent trials on 9 selected datasets with different values of $\rho$ and $\alpha$, combined with different number of dimensions

\begin{tabular}{|c|c|c|c|c|c|c|}
\hline \multirow[t]{2}{*}{$\rho$} & \multirow[t]{2}{*}{$\alpha$} & \multirow[t]{2}{*}{$m$} & \multicolumn{4}{|c|}{ Algorithms } \\
\hline & & & EWKM & LAC & $W$ - $k$-means & PSOVW \\
\hline \multirow[t]{3}{*}{0.5} & \multirow[t]{3}{*}{0.5} & 100 & 75.8 & 71.2 & 71.8 & 89 \\
\hline & & 1000 & 88.2 & 71 & 85.8 & 84 \\
\hline & & 2000 & 74.2 & 67.6 & 75 & 85.6 \\
\hline \multirow[t]{3}{*}{0.5} & \multirow[t]{3}{*}{1} & 100 & 82.8 & 78.2 & 86.6 & 89.2 \\
\hline & & 1000 & 68.8 & 71.2 & 74.4 & 86.4 \\
\hline & & 2000 & 71 & 75.4 & 72.6 & 88.6 \\
\hline \multirow[t]{3}{*}{0.8} & \multirow[t]{3}{*}{1} & 100 & 82.4 & 83 & 86.8 & 85.4 \\
\hline & & 1000 & 69.8 & 77.4 & 72.4 & 85 \\
\hline & & 2000 & 74.2 & 73 & 86.0 & 86.2 \\
\hline
\end{tabular}

and Wolberg 1990) and the insurance company dataset (van der Putten and Someren 2000), which were obtained from the UCI database. ${ }^{3}$ The glass identification dataset consists of 214 instances. Each record has nine numerical variables. The records are classified into two classes: 163 window glass instances and 51 non-window glass instances. Wisconsin breast cancer data has 569 instances with 30 continuous variables. Each record is labeled as benign or malignant. The dataset contains 357 benign records and 212 malignant records. The insurance company dataset has 4000 instances. Each record has 85 numerical variables containing information on customers of an insurance company. A classification label indicating whether the customer is or is not an insurance policy purchaser is provided with each record. The numbers of purchasers and non-purchasers are 3762 and 238, respectively.

\subsection{Results for real data}

To study the effect of the initial cluster centroids, we ran each algorithm ten times on each dataset. Figure 2 presents the average clustering accuracy and the corresponding variance of each algorithm on each real dataset. The horizontal axis represents real datasets. In the left graph, the vertical axis represents the average value of the clustering accuracy for each dataset, and in the right graph it represents the variance of the clustering quality.

From Fig. 2, we can see that PSOVW outperforms the other algorithms. The average clustering accuracy achieved by PSOVW is high on each dataset, while the corresponding variance is very low. PSOVW is a random swarm intelligent algorithm; however, its outputs are very stable. In our experiments, we also observed that other algorithms can achieve good clustering accuracy on the first two datasets on some runs. However, the performances of LAC and EWKM are very unstable on the glass and breast cancer datasets and the performances of the $k$-means and $W$ - $k$-means are unstable on the glass dataset. Their average clustering accuracy is not good, because their iterative procedures are derived from the $k$-means and are very sensitive to the initial cluster centroids. To sum up, PSOVW shows more capability to cluster these datasets and is more stable than other algorithms.

\footnotetext{
${ }^{3}$ http://kdd.ics.uci.edu/.
} 

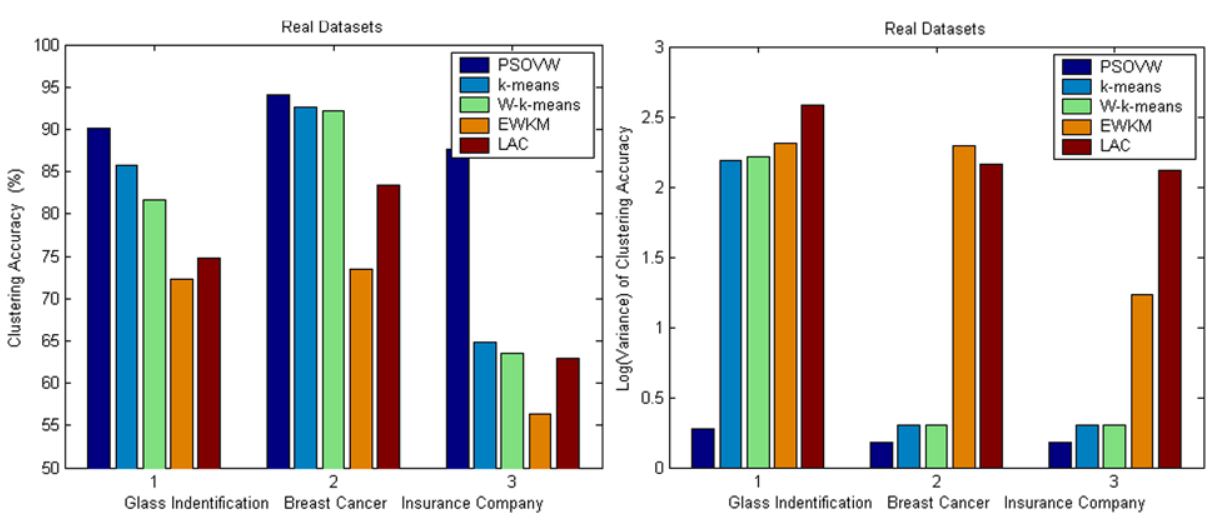

Fig. 2 Average clustering accuracy and the log variance of the clustering accuracy for each algorithm on each real-life dataset

\section{PSOVW application in text clustering}

\subsection{Modifications of PSOVW}

To further demonstrate the efficiency of PSOVW on real data, we apply it to the problem of text clustering, called Text Clustering via Particle Swarm Optimization (TCPSO). To extend PSOVW to text clustering, a few modifications to the algorithm are necessary. In particular, we have adopted the conventional $t f$-idf to represent a text document, and make use of the extended Jaccard coefficient, rather than the Euclidean distance, to measure the similarity between two text documents. Throughout this section, we will use the symbols $n, m$ and $k$ to denote the number of text documents, the number of terms and the number of clusters, respectively. We will use the symbol $L$ to denote the text set, $L_{1}, L_{2}, \ldots, L_{k}$ to denote each one of the $k$ categories, and $n_{1}, n_{2}, \ldots, n_{k}$ to denote the sizes of the corresponding clusters.

In TCPSO, each document is considered as a vector in the term-space. We used the $t f$-idf term weighting model (Salton and Buckley 1988), in which each document can be represented as

$$
\left[t f_{1} \cdot \log \left(n / d f_{1}\right), t f_{2} \cdot \log \left(n / d f_{2}\right), \ldots, t f_{m} \cdot \log \left(n / d f_{m}\right)\right],
$$

where $t f_{i}$ is the frequency of the $i$ th term in the document and $d f_{i}$ is the number of documents that contain the $i$ th term. The extended Jaccard coefficient (Pang-ning et al. 2006) used to measure the similarity between two documents is defined by the following equation:

$$
\operatorname{similarity}(x, z)=\frac{x \cdot z}{\|x\|^{2}+\|z\|^{2}-x \cdot z},
$$

where $x$ represents one document vector, $z$ represents its cluster centroid vector and the symbol ' ' represents the inner product of two vectors. This measure becomes one if two documents are identical, and zero if there is nothing in common between them (i.e., the vectors are orthogonal to each other).

Based on the extended Jaccard coefficient, the weighted similarity between two text documents can be represented as follows:

$$
\frac{\left(w^{\beta} \cdot x\right) \cdot\left(w^{\beta} \cdot z\right)}{\left\|w^{\beta} \cdot x\right\|^{2}+\left\|w^{\beta} \cdot z\right\|^{2}-\left(w^{\beta} \cdot x\right) \cdot\left(w^{\beta} \cdot z\right)},
$$

where $w$ is the weight vector for the corresponding cluster whose centroid is $z$. 
Therefore, the objective function in TCPSO serves to maximize the overall similarity of documents in a cluster and can be modified as follows:

$$
\max F(W)=\sum_{l=1}^{k} \sum_{i=1}^{n}\left(u_{l, i} \cdot \frac{\left(w^{\beta} \cdot x\right) \cdot\left(w^{\beta} \cdot z\right)}{\left\|w^{\beta} \cdot x\right\|^{2}+\left\|w^{\beta} \cdot z\right\|^{2}-\left(w^{\beta} \cdot x\right) \cdot\left(w^{\beta} \cdot z\right)}\right),
$$

where $u$ is the cluster membership of the text document $x$.

\subsection{Text datasets}

To demonstrate the effectiveness of TCPSO in text clustering, we first extract four different structured datasets from 20 newsgroups. ${ }^{4}$ The vocabulary, the documents and their corresponding key terms as well as the dataset are available in http://people.csail.mit.edu/jrennie/ 20 Newsgroups/. We used the processed version of the 20 news-by-date dataset, which is easy to read into Matlab, as a matrix. We also selected four large text datasets from the CLUTO clustering toolkit. ${ }^{5}$ For all datasets, the common words were eliminated using stoplist and all the words were stemmed using Porter's suffix-stripping algorithm (Porter 1980). Moreover, any terms that occur in fewer than two documents were removed.

We chose three famous clustering algorithms (Bisection $k$-means, Agglomeration and Graph) and one soft projected clustering algorithm (EWKM) for comparison with TCPSO. For soft projected clustering algorithms, the similarity between two documents is measured by the extended Jaccard coefficient, while the cosine function is used for the other algorithms, namely Bisection $k$-means, Agglomeration, Graph-based and $k$-means. The second group of text datasets as well as the three clustering algorithms, Bisection $k$-means, Agglomeration and Graph, can be downloaded from the website of CLUTO.

Table 7 presents four different structured text datasets extracted from 20 newsgroups. Each dataset consists of 2 or 4 categories. $n_{i}$ is the number of documents randomly selected from each category. $m$ and $k$ represent the number of terms and the number of categories, respectively. The categories in datasets 2 and 4 are very closely related to each other, i.e., the relevant dimensions of different categories overlap considerably, while the categories in datasets 1 and 3 are highly unrelated, i.e., different categories do not share many relevant dimensions. Dataset 2 contains different numbers of documents in each category.

Table 8 summarizes the characteristics of four large text datasets from CLUTO. To ensure diversity in the datasets, we selected them from different sources. $n, m$ and $k$ are the number of documents, the number of terms and the number of categories, respectively.

Text datasets tr41 \& tr45 are derived from TREC collections (TREC 1999). The categories correspond to the documents relevant to particular queries. Text datasets wap \& $\mathrm{k} 1 \mathrm{~b}$ are from the webACE project (Boley et al. 1999; Han et al. 1998), where each document corresponds to a web page listed in the subject hierarchy of Yahoo.

\subsection{Experimental metrics}

The quality of a clustering solution is determined by three different metrics that examine the class labels of the documents assigned to each cluster. In the following definitions, we

\footnotetext{
${ }^{4}$ http://kdd.ics.uci.edu/.

${ }^{5}$ http://glaros.dtc.umn.edu/gkhome/cluto/cluto/download.
} 
Table 7 The four text datasets extracted from 20 newsgroups
Table 8 The four large text datasets selected from CLUTO

\begin{tabular}{lllll}
\hline Dataset & Source Category & $n_{i}$ & $m$ & $k$ \\
\hline 1 & comp.graphics & 100 & 341 & 2 \\
& alt.atheism & 100 & & \\
2 & talk.politics.mideast & 100 & 690 & 2 \\
& talk.politics.misc & 80 & & \\
3 & comp.graphics & 100 & 434 & 4 \\
& rec.sport.hockey & 100 & & \\
& sci.crypt & 100 & & \\
& talk.religion.misc & 100 & & \\
& alt.atheism & 100 & 387 & \\
& rec.sport.baseball & 100 & & \\
& talk.politics.guns & 100 & & \\
& talk.politics.misc & 100 & & \\
\hline
\end{tabular}

\begin{tabular}{llrrr}
\hline Datasets & Source & $n$ & $m$ & $k$ \\
\hline 5: $\operatorname{tr} 41$ & TREC & 878 & 7454 & 10 \\
6: $\operatorname{tr} 45$ & TREC & 927 & 10128 & 7 \\
7: wap & webACE & 1560 & 8460 & 20 \\
8: k1b & webACE & 2340 & 13879 & 6 \\
\hline
\end{tabular}

Table 9 Experimental metrics

\begin{tabular}{ll}
\hline FScore & $\sum_{r=1}^{k} \frac{n_{r}}{n} \cdot \max _{1 \leq i \leq k} \frac{2 \cdot R\left(L_{r}, S_{i}\right) \cdot P\left(L_{r}, S_{i}\right)}{R\left(L_{r}, S_{i}\right)+P\left(L_{r}, S_{i}\right)}, R\left(L_{r}, S_{i}\right)=\frac{n_{r i}}{n_{r}}, P\left(L_{r}, S_{i}\right)=n_{r i} / m_{i}$ \\
Entropy & $\sum_{i=1}^{k} \frac{m_{i}}{n} \cdot\left(-\frac{1}{\log k} \cdot \sum_{r=1}^{k} \frac{n_{r i}}{n_{i}} \cdot \log \frac{n_{r i}}{m_{i}}\right)$ \\
\hline
\end{tabular}

assume that a dataset with $k$ categories is grouped into $k$ clusters and $n$ is the total number of documents in the dataset. Given a particular category $L_{r}$ of size $n_{r}$ and a particular cluster $S_{i}$ of size $m_{i}, n_{r i}$ denotes the number of documents belonging to category $L_{r}$ that are assigned to cluster $S_{i}$. Table 9 presents the two evaluation metrics used in this paper.

The first metric is the FScore (Zhao and Karypis 2001), which measures the degree to which each cluster contains documents from the original category. In the FScore function, $R$ is the recall value and $P$ is the precision value defined for category $L_{r}$ and cluster $S_{i}$. The second metric is the Entropy (Zhou et al. 2006), which examines how the documents in all categories are distributed within each cluster. In general, FScore ranks the clustering quality from zero (worst) to one (best), while Entropy measures from one (worst) to zero (best). The FScore value will be one when every category has a corresponding cluster containing the same set of documents. The Entropy value will be zero when every cluster contains only documents from a single category. 


\subsection{Experimental results}

\section{Evaluations on text datasets 1-4}

Our first set of experiments focused on evaluating the average quality of the clustering solutions produced by the various algorithms and the influence of text dataset characteristics, such as the number of clusters and the relatedness of clusters, on algorithms. On each smallscale structured text dataset built from 20 newsgroups, we ran the above algorithms 10 times. The average FScore and Entropy values are shown in the following bar graphs. The results of the average FScore values for the six algorithms on datasets 1-4 are shown in the left graph of Fig. 3, while a similar comparison based on the Entropy measure is presented in the right graph.

A number of observations can be made by analyzing the results in Fig. 3. First, TCPSO, Bisection $k$-means and Graph-based yield clustering solutions that are consistently better than those obtained by the other algorithms in all experiments on text datasets $1-4$. This is true whether the clustering quality is evaluated using the FScore or the Entropy measure. These three algorithms produced solutions that are about $5-40 \%$ better in terms of FScore and around 5-60\% better in terms of Entropy.

Second, TCPSO yielded the best solutions irrespective of the number of clusters, the relatedness of clusters and the measure used to evaluate the clustering quality. Over the first set of experiments, the solutions achieved by TCPSO are always the best. On average, TCPSO outperforms the next best algorithm, Graph-based, by around 3-9\% in terms of FScore and 9-21\% in terms of Entropy. In general, it achieves higher FScore values and lower Entropy values than the other algorithms. The results in the FScore graph are in agreement with those in the Entropy graph. Both of them show that it greatly surpasses other algorithms for text clustering.

In general, the performances of TCPSO, Graph-based and Bisection $k$-means deteriorate on text datasets where categories are highly related. In such documents, each category has a subset of words and two categories share many of the same words. Basically, the probability of finding the true centroid for a document varies from one dataset to another, but it decreases as category relatedness increases. Thus, we would expect that a document can often fail to yield the right centroid regardless of the metric used.

Third, except for the Entropy measure on text datasets 1 and 4, Graph-based performs the next best for both FScore and Entropy. Bisection $k$-means also performs quite well when the
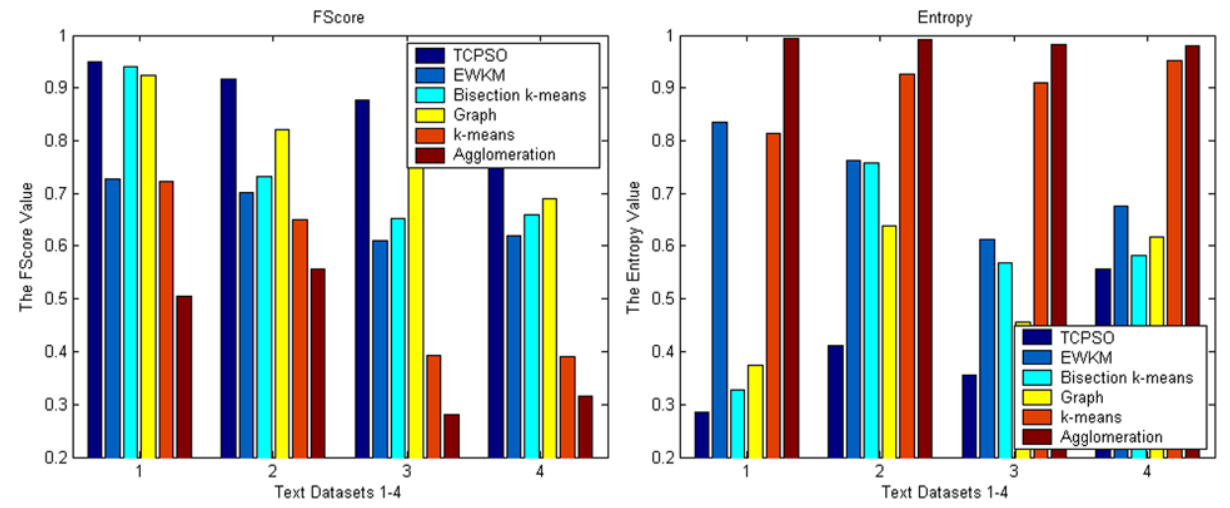

Fig. 3 FScore and Entropy values of each algorithm on datasets 1-4 
categories in datasets are highly unrelated. EWKM always performs in the middle range, due to its employment of the local search strategy. The solutions yielded by the $k$-means fluctuate in all experiments. Agglomeration performs the worst of the six algorithms, yielding small FScore and large Entropy values on all datasets. This means the number of documents from each cluster yielded by Agglomeration is thus more balanced in one genuine category and as a result, the number of documents that are correctly identified by Agglomeration in the genuine category is very low.

\section{Evaluations on text datasets 5-8}

To further investigate the behavior of TCPSO, we performed a second series of experiments, focused on evaluating the comprehensive performance of each algorithm on large text datasets. Each algorithm was independently run 10 times on each of datasets 5-8. The average FScore values for the six algorithms on datasets 1-4 are shown in the left graph of Fig. 4, while a similar comparison based on the Entropy measure is presented in the right graph.

Looking at the two graphs of Fig. 4 and comparing the performance of each algorithm on datasets 5-8, we can see that TCPSO outperformed all other algorithms, on average, although Graph-based and Bisection $k$-means are very close. Graph-based and Bisection $k$-means are respectively worse than TCPSO in terms of FScore by $1-11 \%$ and $6-10 \%$, on average, while they yield similar Entropy values to TCPSO. That means each category of documents is mainly distributed within its dominant cluster and TCPSO recovers more documents of each cluster primarily from their original categories.

Although Graph-based and Bisection $k$-means performed quite well on the datasets $\operatorname{tr} 41$ and $\mathrm{k} 1 \mathrm{~b}$, their performances deteriorate sharply on the datasets tr $45 \&$ wap, where there is considerable overlap between the key words of different categories, such as the categories cable and television, multimedia and media etc. They failed to perform well on such datasets, while TCPSO still achieved relatively high FScore values because it indentifies clusters embedded in subspaces by iteratively assigning an optimal feature weight vector to each cluster. The other three algorithms occasionally work well, achieving FScore values above 0.8 on the dataset $\mathrm{k} 1 \mathrm{~b}$, whose categories (business, politics, sports etc.) are highly unrelated, but the FScore values are relatively low on other datasets. Moreover, their corresponding Entropy values are very high, which means each category of documents is more evenly distributed within all the clusters.
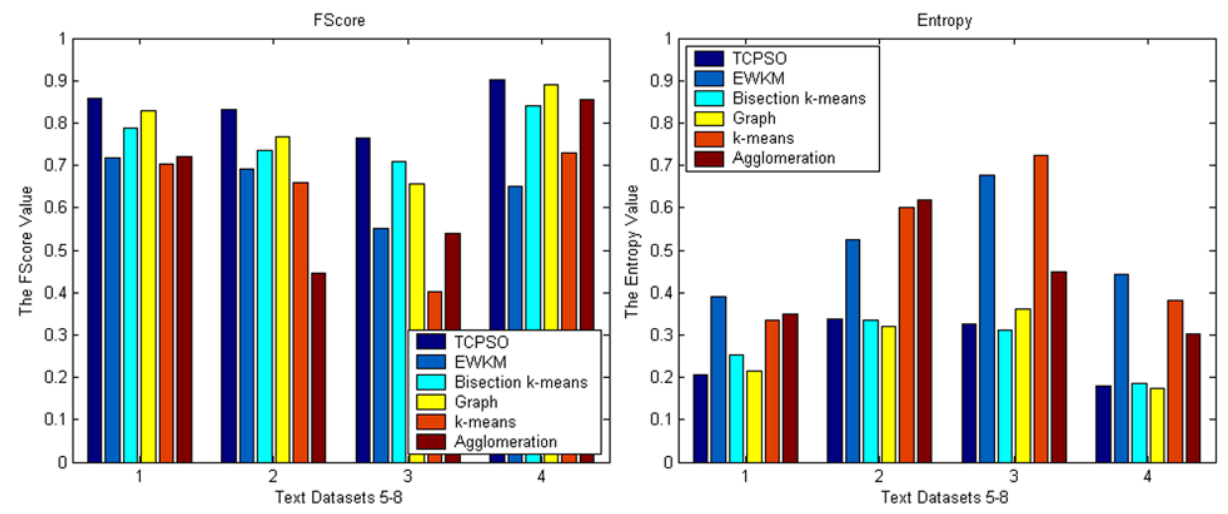

Fig. 4 Average FScore and Entropy values of each algorithm on datasets 5-8 


\section{Conclusions}

In response to the needs arising from the emergence of high-dimensional datasets in data mining applications and the low cluster quality of projected clustering algorithms, this paper proposed a particle swarm optimizer for the variable weighting problem, called PSOVW. The selection of the objective function and the search strategy is very important in soft projected clustering algorithms. PSOVW employs a $k$-means weighting function, which calculates the sum of the within-cluster distance for each cluster along relevant dimensions preferentially to irrelevant ones. It also proposes a normalized representation of variable weights in the objective function, which greatly facilities the search process. Finally, the new algorithm makes use of particle swarm optimization to get near-optimal variable weights for the given objective function. Although PSOVW runs more slowly than other algorithms, its deficiency in running time is acceptable. Experimental results on synthetic and real-life datasets, including an application to document clustering, show that it can greatly improve clustering accuracy for high-dimensional data and is much less sensitive to the initial cluster centroids. Moreover, the application to text clustering shows that the effectiveness of PSOVW is not confined to Euclidean distance. The reason is that the weights are only affected by the values of the objective function and weight updating in PSOVW is independent of the similarity measure. Other similarity measures can thus be used for different applications: the extended Jaccard coefficient for text data, for instance.

Two issues need to be addressed by the PSOVW algorithm. PSOVW is still not able to recover the relevant dimensions totally. In fact, none of the tested algorithms, including PSOVW, consistently identify the relevant dimensions to achieve their final clustering results; i.e., some irrelevant dimensions may have high weights while some relevant dimensions may have low weights. The dimensionality of the datasets and the number of relevant dimensions for each cluster are extremely high compared to the number of data points, resulting in very sparse datasets. This might be the main reason why these algorithms fail to recover all the relevant dimensions. Further investigations are needed. The other major issue in PSOVW is that usually the structure of the data is completely unknown in real-world data mining applications and it is thus very difficult to provide the number of clusters $k$. Although a number of different approaches for determining the number of clusters automatically have been proposed (Tibshirani and Walther 2000; Handl and Knowles 2004), no reliable method exists to date, especially for high-dimensional clustering. In the Ph.D. work of the first author, Yanping (2009), the PSOVW has been extended to deal also with the problem of automatically determining the number of clusters $k$.

Acknowledgements We are thankful to Prof. Suganthan for providing the Matlab coding of CLPSO. We thank Tengke Xiong and Yassine Parakh Ousman for their help with the DiSH and HARP algorithms. We also thank the anonymous reviewers for their constructive comments on our manuscript.

\section{Appendix}

The algorithm for generating a synthetic dataset is derived from but not identical to the one presented in Jing et al. (2007).

- Specify the number of clusters $k$, the number of dimensions $m$ and the number of objects $n$. Set three parameters, $\varepsilon, \rho$ and $\alpha$. 
- // Determine relevant dimensions for each cluster.

// Determine the number of relevant dimensions $m_{l}$ for each cluster.

For each cluster $l$,

Assign a random number $m_{l} \in[2, m]$, where $\sum m_{l}=\varepsilon * k * m$.

For each cluster $l$,

If $l==1$,

Randomly choose $m_{1}$ relevant dimensions for $C_{1}$.

Otherwise,

Randomly choose $\rho * m_{l}$ relevant dimensions from the relevant dimensions of $C_{l-1}$.

Randomly choose $(1-\rho) * m_{l}$ relevant dimensions from the other dimensions. End.

- //Generate the mean $u$ and the variance $\sigma$ for each relevant dimension of each cluster.

$\sigma=1$

For each cluster $l$,

If the relevant dimension $j$ is not a common relevant dimension of clusters $C_{l}$ and

$C_{l-1}$,

Randomly set $u_{l, j}$ in $[0,100]$.

Otherwise,

If $\left(u_{l-1, j}+\alpha * \sigma\right)>100$,

$u_{l, j}=u_{l-1, j}-\alpha * \sigma$.

Otherwise,

End.

$u_{l, j}=u_{l-1, j}+\alpha * \sigma$.

End.

- //Generate data points for each cluster.

For each cluster $l$,

//Specify the number of points $n_{l}$.

$n_{l}=n / k$;

For each dimension $j$,

If $j$ is a relevant dimension of $C_{l}$,

Produce the data points with a normal distribution, $N\left(u_{l, j}, \sigma\right)$.

Otherwise,

Uniformly produce the data points in $[0,10]$.

End.

\section{References}

Agrawal, R., Gehrke, J., Gunopulos, D., \& Raghavan, P. (2005). Automatic subspace clustering of high dimensional data. Data Mining and Knowledge Discovery, 11(1), 5-33.

Aitnouri, E., Wang, S., \& Ziou, D. (2000). On comparison of clustering techniques for histogram $p d f$ estimation. Pattern Recognition and Image Analysis, 10(2), 206-217.

Boley, D., Gini, M., et al. (1999). Document categorization and query generation on the world wild web using WebACE. AI Review, 11, 365-391.

Bouguessa, M., Wang, S., \& Sun, H. (2006). An objective approach to cluster validation. Pattern Recognition Letters, 27(13), 1419-1430.

Domeniconi, C., Gunopulos, D., Ma, S., Yan, B., Al-Razgan, M., \& Papadopoulos, D. (2007). Locally adaptive metrics for clustering high dimensional data. Data Mining and Knowledge Discovery Journal, 14, 63-97. 
Eberhart, R. C., \& Kennedy, J. (1995). A new optimizer using particle swarm theory. In Proc. 6th international symposium on micromachine and human science, Japan (pp. 39-43).

Elke, A., Christian, B., et al. (2008). Detection and visualization of subspace cluster hierarchies. In $L N C S$ (Vol. 4443, pp. 152-163). Berlin: Springer.

Evett, I. W., \& Spiehler, E. J. (1987). Rule induction in forensic science. Central Research Establishment. Home Office Forensic Science Service, Aldermaston, Reading, Berkshire RG7 4PN.

Goil, G. S., Nagesh, H., \& Choudhary, A. (1999). Mafia: Efficient and scalable subspace clustering for very large data sets. Technical Report CPDC-TR-9906-010, Northwestern University.

Han, E. H., Boley, D., et al. (1998). WebACE: A web agent for document categorization and exploration. In Proc. of 2 nd international conf. on autonomous agents.

Handl, J., \& Knowles, J. (2004). Multiobjective clustering with automatic determination of the number of clusters. Technical Report, UMIST, Department of Chemistry.

Huang, J. Z., Ng, M. K., Rong, H., \& Li, Z. (2005). Automated dimension weighting in $k$-means type clustering. IEEE Transactions on Pattern Analysis and Machine Intelligence, 27(5), 1-12.

Jing, L., Ng, M. K., \& Huang, J. Z. (2007). An entropy weighting $k$-means algorithm for subspace clustering of high-dimensional sparse data. IEEE Transactions on Knowledge and Data Engineering, 19(8), 10261041.

Kriegel, H.-P., Kroger, P., \& Zimek, A. (2009). Clustering high-dimensional data: A survey on subspace clustering, pattern-based clustering, and correlation clustering. ACM Transactions on Knowledge Discovery Data, 3(1), Article 1.

Liang, J. J., Qin, A. K., Suganthan, P. N., \& Baskar, S. (2006). Comprehensive learning particle swarm optimizer for global optimization of multimodal functions. IEEE Transactions on Evolutionary Computation, 10(3), 281-295.

Lu, Y. (2009). Particle swarm optimizer: applications in high-dimensional data clustering. Ph.D. Dissertation, University of Sherbrooke, Department of Computer Science.

Makarenkov, V., \& Legendre, P. (2001). Optimal variable weighting for ultrametric and additive trees and $k$-means partitioning: Methods and software. Journal of Classification, 18(2), 245-271.

Mangasarian, O. L., \& Wolberg, W. H. (1990). Breast cancer diagnosis via linear programming. SIAM News, 23(5), 1-18.

Moise, G., \& Sander, J. (2008). Finding non-redundant, statistically significant regions in high dimensional data: A novel approach to projected and subspace clustering. In Proc. of the 14th ACM SIGKDD international conference on knowledge discovery and data mining (pp. 533-541).

Moise, G., Sander, J., \& Ester, M. (2008). Robust projected clustering. Knowledge and Information Systems, 14(3), 273-298.

Onwubolu, G. C., \& Clerc, M. (2004). Optimal operating path for automated drilling operations by a new heuristic approach using particle swarm optimization. International Journal of Production Research, 42(3), 473-491.

Pang-ning, T., Michael, S., \& Vipin, K. (2006). Introduction to data mining (p. 77). Upper Saddle River: Pearson Education.

Parsons, L., Haque, E., \& Liu, H. (2004). Subspace clustering for high dimensional data: A review. SIGKDD Explorations Newsletter, 6, 90-105.

Porter, M. F. (1980). An algorithm for suffix stripping. Program, 14(3), 130-137.

Procopiuc, C. M., Jones, M., Agarwal, P. K., \& Murali, T. M. (2002). A Monte Carlo algorithm for fast projective clustering. In Proc. of ACM SIGMOD international conference on management of data (pp. 418427).

Salman, A., Ahmad, I., \& Al-Madani, S. (2003). Particle swarm optimization for task assignment problem. Microprocessors and Microsystems, 26(8), 363-371.

Salton, G., \& Buckley, C. (1988). Term-weighting approaches in automatic text retrieval. Information Processing \& Management, 24(5), 513-523.

Tasgetiren, M. F., Sevkli, M., Liang, Y.-C., \& Gencyilmaz, G. (2004). Particle swarm optimization algorithm for single machine total weighted tardiness problem. In Proc. of the 2004 congress on evolutionary computation (CEC'04), Portland, Oregon (pp. 1412-1419).

TREC (1999). Text retrieval conference, http://trec.nist.gov/.

Tibshirani, R., Walther, G., et al. (2000). Estimating the number of clusters in a dataset via the Gap Statistic. Technical Report, Stanford Univeristy.

Van den Bergh, F., \& Engelbecht, A. P. (2000). Cooperative learning in neural networks using particle swarm optimizers. South African Computer Journal, 26, 84-90.

van der Putten, P., \& van Someren, M. (Eds.) (2000). CoIL challenge 2000: the insurance company case. Published by Sentient Machine Research, Amsterdam. Technical Report.

Woo, K.-G., Lee, J.-H., Kim, M.-H., \& Lee, Y.-J. (2004). FINDIT: A fast and intelligent subspace clustering algorithm using dimension voting. Information and Software Technology, 46(4), 255-271. 
Yoshida, H., Kawata, K., Fukuyama, Y., \& Nakanishi, Y. (2000). A particle swarm optimization for reactive power and voltage control considering voltage security assessment. IEEE Transactions on Power Systems, 15(4), 1232-1239.

Zhao, Y., \& Karypis, G. (2001). Criterion functions for document clustering: Experiments and analysis. Technical Report, CS Dept., Univ. of Minnesota.

Zhou, X., Hu, X., Zhang, X., Lin, X., \& Song, I.-Y. (2006). Context-sensitive semantic smoothing for the language modeling approach to genomic IR. In SIGIR'06. 